Research Article

\title{
Study of Working Medium Performance by Acoustic Emission in EDM Machining of Ti6Al4V
}

\author{
Chengbo Guo $\mathbb{D}^{1},{ }^{1}$ Shufa Sun, ${ }^{1}$ Shichun $\mathrm{Di}^{2}{ }^{2}$ and Dongbo Wei ${ }^{2}$ \\ ${ }^{1}$ School of Engineering and Technology, Northeast Forestry University, Harbin 150040, China \\ ${ }^{2}$ School of Mechatronics Engineering, Harbin Institute of Technology, Harbin 15001, China \\ Correspondence should be addressed to Chengbo Guo; chengbo.guo@nefu.edu.cn
}

Received 18 August 2020; Revised 23 September 2020; Accepted 24 October 2020; Published 3 November 2020

Academic Editor: Lenka Zajičková

Copyright ( $\odot 2020$ Chengbo Guo et al. This is an open access article distributed under the Creative Commons Attribution License, which permits unrestricted use, distribution, and reproduction in any medium, provided the original work is properly cited.

In electrical discharge machining (EDM), the working medium plays an important role in the material removal process. Lots of methods have been utilized to study this process, but a widely accepted explanation about this process has not been yet accomplished. In this study, the acoustic emission (AE) sensor was fixed on EDM machine to study the material removal process by observing the expansion and contraction process of gas bubble surrounding the discharge plasma. The machining performance in different working mediums was studied for Ti-6Al-4V machining in air, kerosene, and water-based emulsion. Discharge in different working mediums would result in different material removal rates and surface quality. The difference of AE wave frequency domain distribution for discharge in different working mediums was studied. It was observed that the frequency of acoustic emission wave generated by discharge in different working mediums would be different. The characteristic difference of single AE wave generated by discharge in air, kerosene, and water-based emulsion was compared. It was found that the duration time and peak amplitude of acoustic emission wave generated by discharge in different working mediums were different, and the acoustic emission wave generated by discharge in water-based emulsion would last longer and get higher peak amplitude compared to discharge in air and kerosene. The significant difference of AE wave generated by discharge in water-based emulsion from that in kerosene was found. Based on the acoustic emission wave observation, the special characteristic of the material removal process for discharge in water-based emulsion was found.

\section{Introduction}

It has been reported that electrical discharge machining (EDM) has great application potential in aerospace industry, especially for titanium alloys and nickel-based superalloy machining $[1,2]$. In electrical discharge machining, material removal was realized by a short and complicated physical reaction process, which takes place around a high-temperature plasma column initialed by electrical discharge in the working medium [3]. During the discharge process, strong light radiation was generated around the discharge channel, which made the observation of the material removal process challenging [4].

It was found that the working medium has a significant impact on the material removal process [5-7]. Many researchers studied the relationship between discharge generated bubbles and discharge locations $[8,9]$. Kitamura et al. took a transparent $\mathrm{SiC}$ single crystal as the tool electrode to observe the distribution of bubbles and discharge locations in the discharge gap using a high-speed video camera $[10,11]$. They found that the radial flow and bubble oscillation difference make the material removal rate in the water higher than that of oil. Schulze et al. studied the propagation of gas bubble during the discharge process using high-speed frame camera $[12,13]$; it was observed that a higher discharge current and longer pulse duration time would be beneficial to the bubble propagation process.

For the purpose of analyzing the mechanism of material removal in EDM, Hayakawa et al. studied the relationship between debris particles moving tracks and the bubble oscillation process [14]. They found that the material removal only occurred during the bubble expansion process. In order 
to understand the mechanism of the debris exclusion process in EDM, Wang et al. established an experimental setup by using transparent hot-melted EVA material covered on electrode [15]. It was observed that the expansion motion of the bubble was the main factor that excludes the melted materials from the discharge area.

Many models have been established for the material removal process $[16,17]$. Yang et al. studied the crater formation process by molecular dynamics simulation [18]; the result showed that the melted material was removed in the form of clusters by the explosion of the bubble. Based on dynamic simulation and experiment, Liu et al. investigated the material removal process by studying energy distribution and working medium. It was observed that a smaller interelectrode distance would result in a higher pressure bubble in the discharge gap, which was helpful to the melted material removal process [19]. The material removal process can also be improved by adopting dielectric with higher viscosity as a working medium $[20,21]$.

Tamura et al. studied the crater formation process by measuring the impulsive forces during the discharge process [22]. They found that the expansion and contraction of the generated bubble caused the impulsive force during the discharge process, but the influence of the impulsive force on crater formation was insignificant. Natsu et al. measured the diameter and temperature of arc plasma during the discharge process using spectroscopy [23-25]. It was observed that the plasma expanding process finished in $2 \mu \mathrm{s}$ after discharge breakdown and the diameter of the formed crater was larger than that of the arc plasma.

It can be observed from the above research results that the expansion and extraction of the bubble around the discharge channel have an apparent influence on the material removal process, but its relationship has not been yet figured out. Acoustic emission (AE) was used to observe the rapid release of energy from a limited area [26, 27]. It can be applied to observe the energy release during the discharge process in EDM machining. This research aims to study the relationship between bubble oscillation motion and the material removal process. The AE sensor was utilized to detect the pressure oscillation of gas bubble formed by the discharge process. Air, kerosene, and water-based emulsion were adopted as working mediums to study the influence of gas bubble pressure oscillation on the material removal process. The expansion and contraction frequency of gas bubble surrounding the discharge plasma was studied based on the frequency domain distribution analysis. The phenomenon that discharge in water-based emulsion would generate a longer duration time $\mathrm{AE}$ wave with two peaks was found, and its mechanism was discussed based on the analysis of the discharge process.

\section{Materials and Methods}

To figure out the difference in the material removal process in different working mediums, the relationship between gas bubble development process and the material removal process was studied through collecting the AE wave generated during gas bubble expansion and contraction process.
The experiment setup is illustrated in Figure 1. The AE sensor (Kistler AE-8152B2) was placed in the working medium to collect the AE wave generated during the discharge process. In order to eliminate the influence of the $\mathrm{AE}$ sensor position on the strength of the collected AE wave, the distance of AE sensor to discharge point was set at $8 \mathrm{~mm}$ in both horizontal and vertical directions.

The experiments were conducted on AGIETRON 2 EDM machine, and the electrode feeding speed would autoadjust based on the discharge process. The weight of the workpiece was measured using METTLER TOLEDO ME104 electronic balance before and after machining. The surface topography of the machined workpiece was detected using Dino-Lite AM4515T8 microscope. The graphite was selected as the tool electrode. Titanium alloy Ti-6Al-4V which is a typical difficult-to-cut material $[28,29]$ was chosen as the workpiece. Air, kerosene, and water-based emulsion were selected as the working medium to make a comparison. The same working conditions were applied to all different working mediums. The distance and angle of the AE sensor to the discharge area were also fixed in all the tests.

The AE sensor was sensitive to the pressure oscillation of the work medium caused by the fierce expansion and contraction of gas bubble volume surrounding the plasma during the discharge process. Both $\mathrm{AE}$ wave and discharge voltage change during the discharge process were collected simultaneously. The frequency domain distribution of $\mathrm{AE}$ wave was analyzed based on fast Fourier transform (FFT). The details of AE wave in different stages were studied in accordance with the discharge voltage change.

To study the electrical discharge machining performance in different working mediums, titanium alloy Ti6Al4V was machined in air, kerosene, and water-based emulsion, respectively, under the machining condition as listed in Table 1. During the discharge process, both electrode and workpiece were immersed in the working medium. Considering the AE wave would last hundreds of microseconds during the discharge process, the pulse-on time was set at $100 \mu$ s with a pulse-off time of $1000 \mu \mathrm{s}$ in order to collect a complete AE wave.

The material removal rate (MRR) is calculated by

$$
\operatorname{MRR}=\frac{(W / \rho)}{t}
$$

where $W(g)$ is the weight of the removed material, $\rho\left(\mathrm{mm}^{3} /\right.$ $\mathrm{min})$ is the density of workpiece, and $t(\mathrm{~min})$ is the machining time.

\section{Results and Discussion}

3.1. Machining Performance Comparison in Different Working Media. In order to study the influence of the working medium property on machining performance, Ti-6Al-4V was machined in air, kerosene, and graphite, respectively. The MRR and machined surface were compared. The MRR difference in different working mediums is shown in Figure 2; it can be noticed that the MRR for discharge in air was limited. It indicates that the melted material by the discharge process could not be removed from the workpiece which 


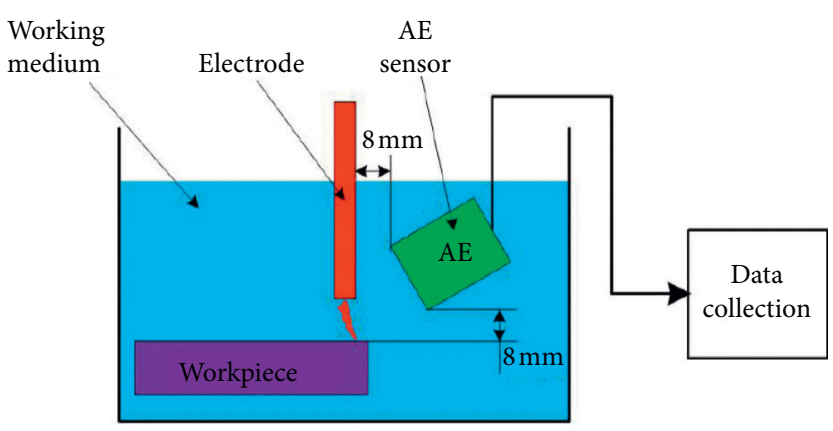

(a)

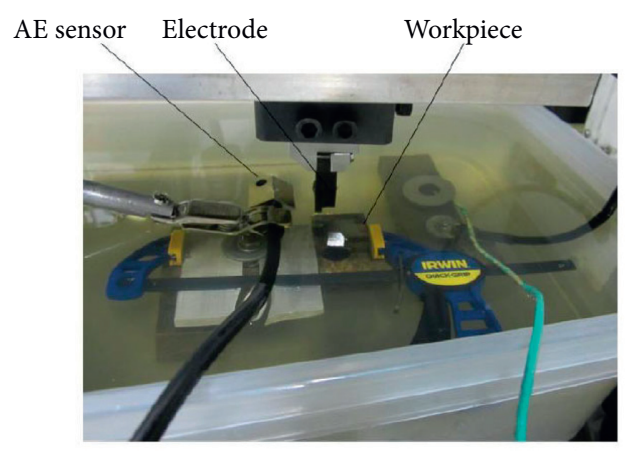

(b)

FIGURE 1: The placement of the experiment setup: (a) schematic diagram and (b) experiment setup.

TABle 1: Process parameters for working medium tests.

\begin{tabular}{lc}
\hline Process parameters & Settings \\
\hline Pulse-on time $(\mu \mathrm{s})$ & 100 \\
Pulse-off time $(\mu \mathrm{s})$ & 1000 \\
Current $(\mathrm{A})$ & 8 \\
Open gap voltage $(\mathrm{V})$ & 180 \\
Tool electrode polarity & Negative \\
Working medium & Air, kerosene, water-based emulsion (5\%) \\
Electrode material & Graphite \\
Workpiece material & Ti-6Al-4V \\
\hline
\end{tabular}

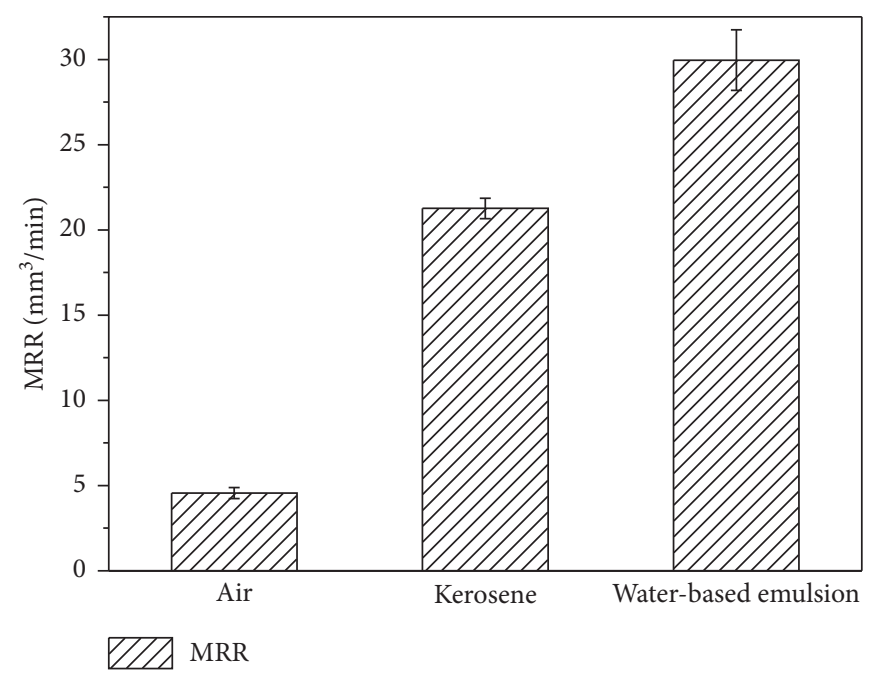

FIGURE 2: MRR comparison in 3 different working mediums.

would resolidify on the workpiece surface after the discharge process. The discharge in kerosene and water-based emulsion can obtain a higher MRR than discharge in air. The MRR in the water-based emulsion was higher than that in kerosene by $41 \%$. Discharge in water-based emulsion has a remarkable material removal performance compared with kerosene under the same machining conditions. It demonstrates that the working medium has a significant influence on the material removal process.
Figure 3 shows the surface difference of EDM machined Ti6Al4V in different working mediums under the machining condition as listed in Table 1 . It can be observed that the machined surface in different working mediums has a significant difference. The surface machined in air turned white. It indicates that discharge in air has a poor cooling performance which leads the workpiece surface to be oxidized in high temperature. Compared with surface machined in kerosene, the surface machined in water-based 


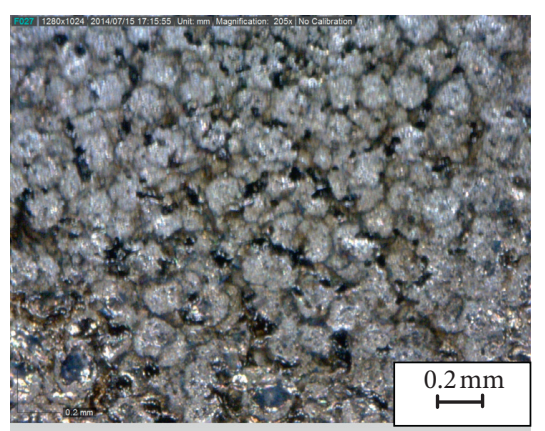

(a)

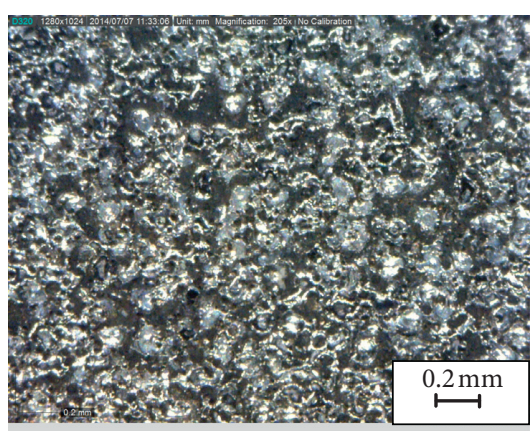

(b)

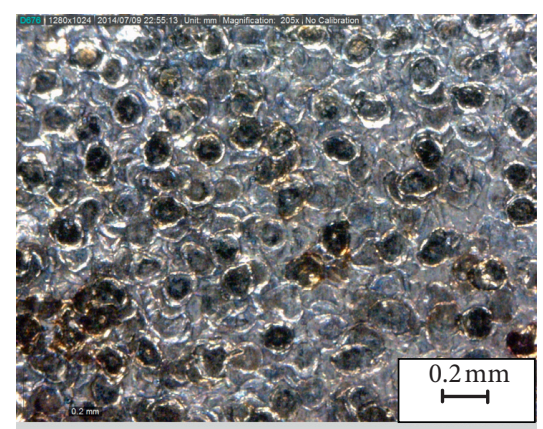

(c)

FIGURE 3: EDM machined surface comparison in 3 different working mediums: (a) discharge in air; (b) discharge in kerosene; and (c) discharge in water-based emulsion.

emulsion would be rougher and covered with cylindrical pits. It demonstrates that the machined surface quality has a direct relationship with the property of the working medium.

In order to study the difference of discharge process in different working mediums, the bubble oscillation phenomenon was investigated by collecting the $\mathrm{AE}$ wave emitted by the discharge process. The frequency domain distribution of AE wave emitted by the electrical discharge process in air, kerosene, and water-based emulsion is shown in Figure 4. It can be observed that the $\mathrm{AE}$ wave generated by discharge in air was weak, and its frequency was mainly distributed between $100 \mathrm{kHz}$ and $200 \mathrm{kHz}$. The intensity of the $\mathrm{AE}$ wave generated by the discharge in kerosene was higher than that in the air, its frequency distribution was mainly distributed between $230 \mathrm{kHz}$ and $280 \mathrm{kHz}$, and the strongest AE signal is with the frequency of $251 \mathrm{kHz}$. Compared to discharge in air and kerosene, the strength of the $\mathrm{AE}$ wave generated by the discharge in water was stronger, and its frequency was mainly distributed between $230 \mathrm{kHz}$ and $320 \mathrm{kHz}$. There were two concentrated AE signal frequencies distributed around $263 \mathrm{kHz}$ and $288 \mathrm{kHz}$ for discharge in water-based emulsion. According to the frequency domain distribution analysis, the pressure oscillation frequency was influenced by the working medium, which indicated that the expansion and contraction process of gas bubble surrounding the discharge plasma was influenced by the property of the working medium. Therefore, the material removal performance in different working mediums can be studied based on the analysis of the AE phenomenon during the discharge process.

In order to study the details of the AE wave during the discharge process, the single discharge process in air, kerosene, and water-based emulsion was collected by $\mathrm{AE}$ sensor, respectively; the result is listed in Figures 5-7. The AE wave generated by the electrical discharge in air is shown in Figure 5. It can be noticed that the AE generated by discharge in air was mainly triggered by the discharge breakdown process, and its strength was very weak. This phenomenon indicated that there was no fierce pressure oscillation happening in the gap for discharge in air, which was not conducive to the material removal process and result in a limited MRR. It illustrates that the fierce pressure oscillation surrounding discharge plasma is important for effective material removal.

Figure 6 shows the AE wave generated by discharge in kerosene. It can be noticed that the amplitude of $\mathrm{AE}$ wave was higher than that in air, which indicated that the discharge released energy intensity in kerosene was higher than that in air. The strongest AE signal was detected at the discharge breakdown moment. After that, the AE signal became weak as the discharge process continues. It demonstrated that the maximum pressure fluctuation happened as a result of the discharge breakdown process. During this process, a high-pressure gas bubble was formed by the hightemperature discharge plasma in a short time, which resulted in the generation of $\mathrm{AE}$ wave. The pressure oscillation caused by the expansion and contraction process of gas bubble surrounding discharge plasma was influenced by the property of kerosene. In accordance with the result of Figures 2 and 3, higher energy intensity with fierce pressure oscillation in discharge area would be beneficial to the material removal process. Discharge in liquid would generate a stronger AE wave than that in air.

The AE wave generated by water-based emulsion is shown in Figure 7. Compared with discharge in kerosene, the $\mathrm{AE}$ wave generated by discharge in water-based emulsion would have a longer duration time with two apparent peaks. It demonstrated that the energy intensity released by discharge in the water-based emulsion was higher than that in kerosene. The first peak was detected at the discharge breakdown moment like that in kerosene, and the second peak was detected after the end of the discharge process. This phenomenon indicated that the fierce pressure oscillation happened at the discharge breakdown moment and after the end of discharge, which means the expansion and contraction process of gas bubble surrounding discharge plasma would be continued for a certain time. A reasonable explanation is that the water surrounding the discharge plasma channel is decomposed into hydrogen and oxygen by high temperatures produced during the discharge process. At the end of discharge, the combustion of hydrogen and oxygen in a limited space causes a drastic pressure drop in the gap, which results in a cavitation effect and generated a strong $\mathrm{AE}$ 


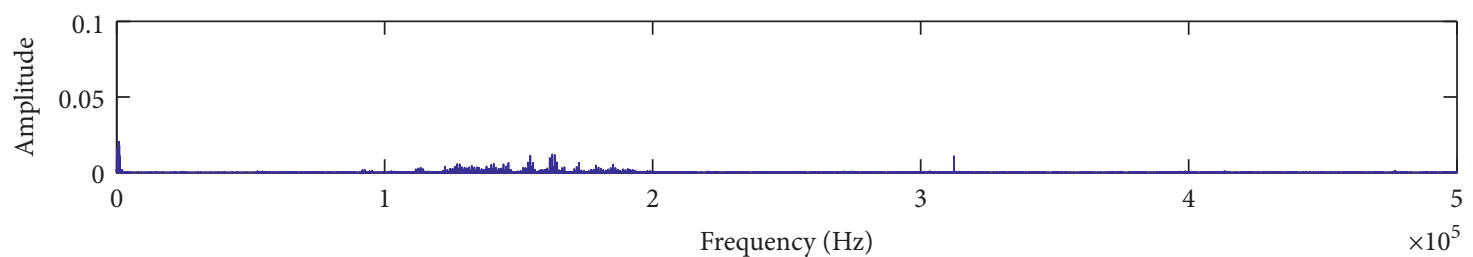

(a)

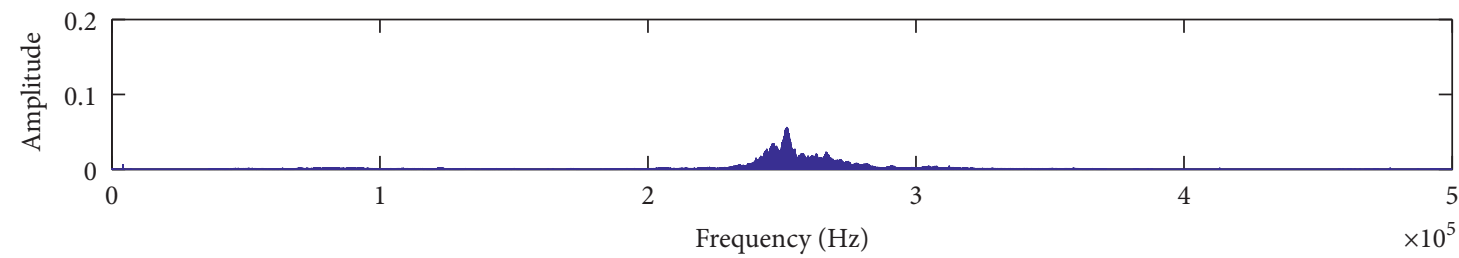

(b)

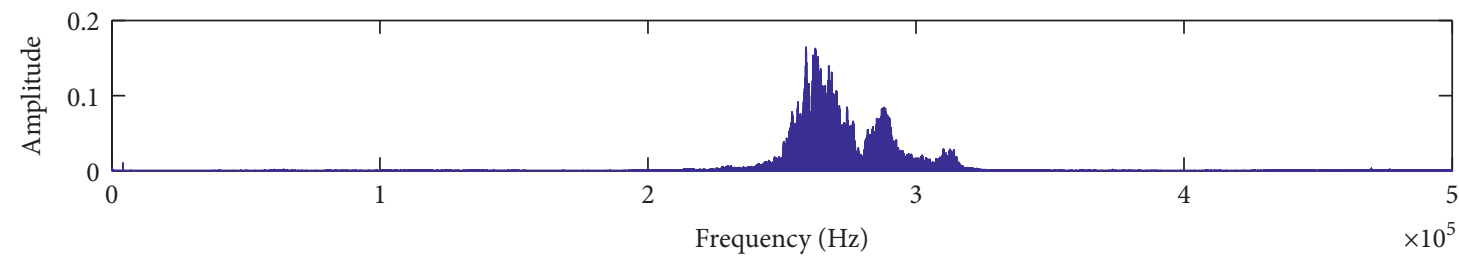

(c)

FIGURE 4: The FFT of AE signal by discharge in different working mediums: (a) for discharge in air; (b) for discharge in kerosene; and (c) for discharge in water-based emulsion. Acoustic emission analysis in different working mediums.

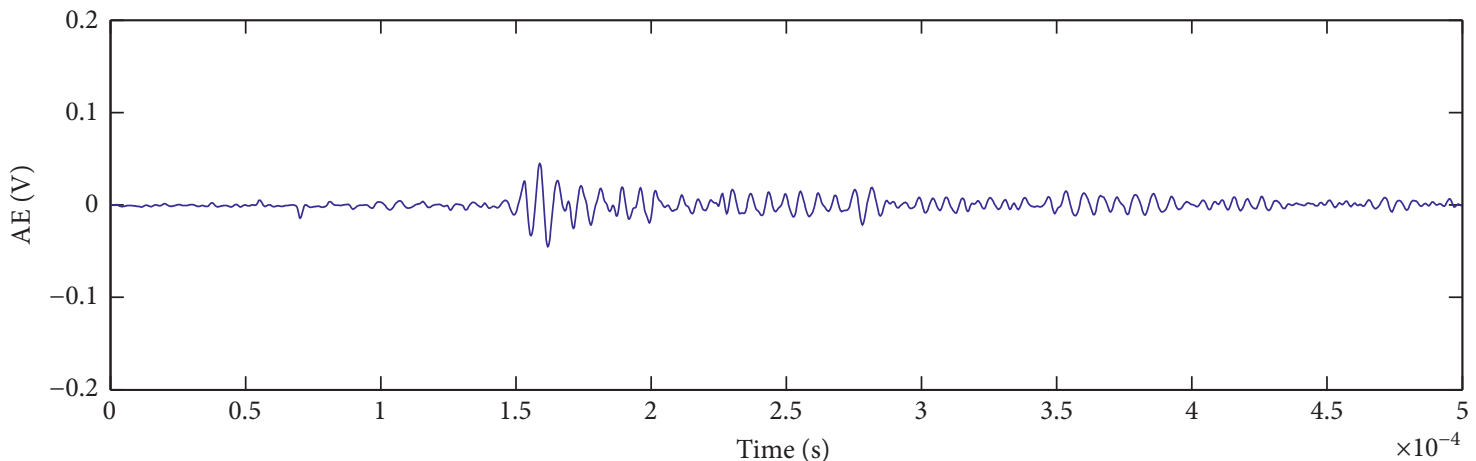

(a)

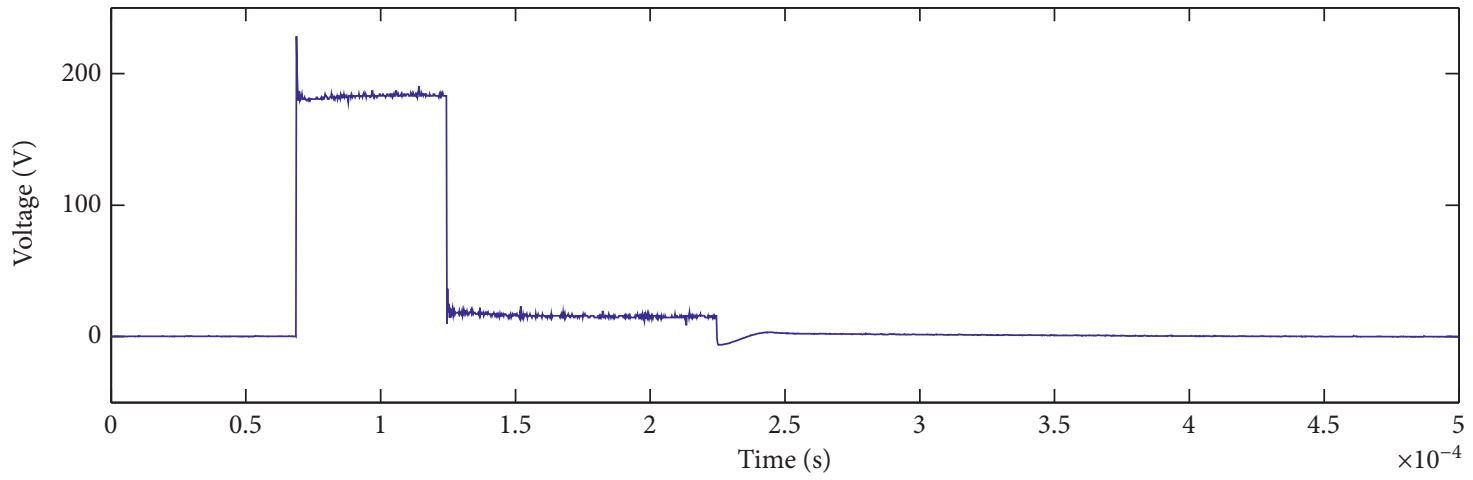

(b)

Figure 5: AE wave generated by discharge in air: (a) AE signal and (b) discharge voltage. 


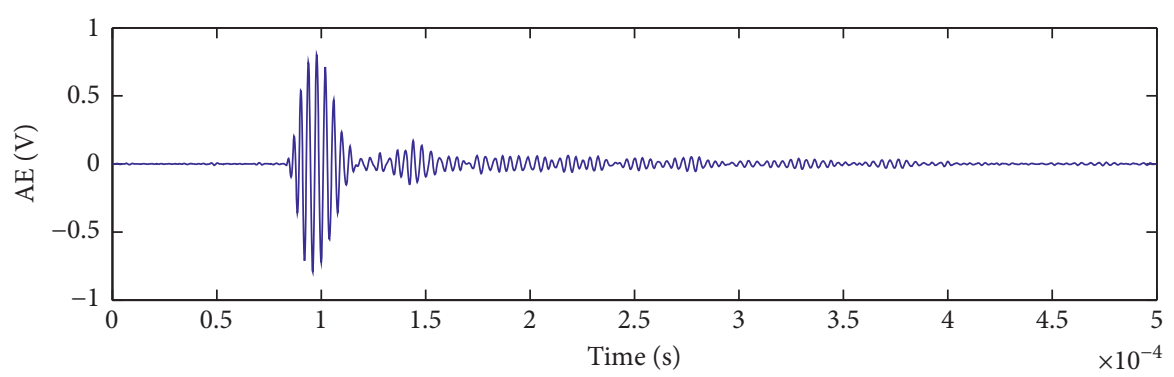

(a)

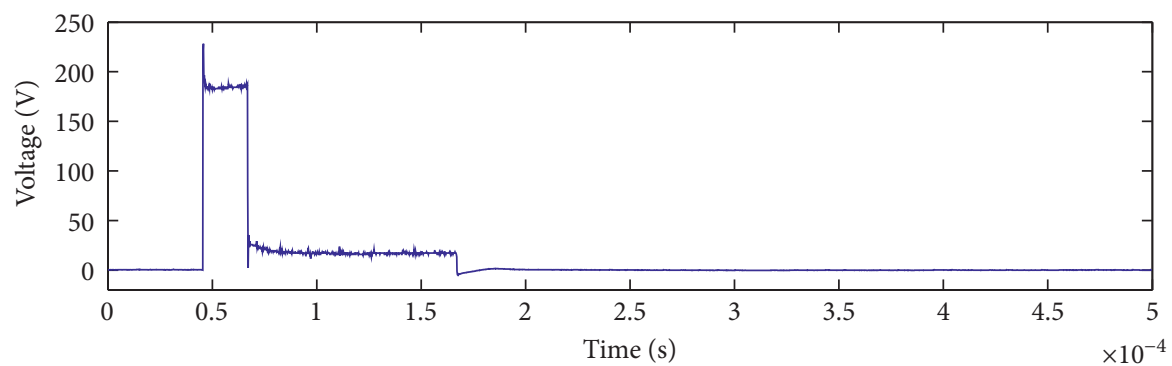

(b)

FIgURE 6: AE wave generated by discharge in kerosene: (a) AE signal and (b) discharge voltage.

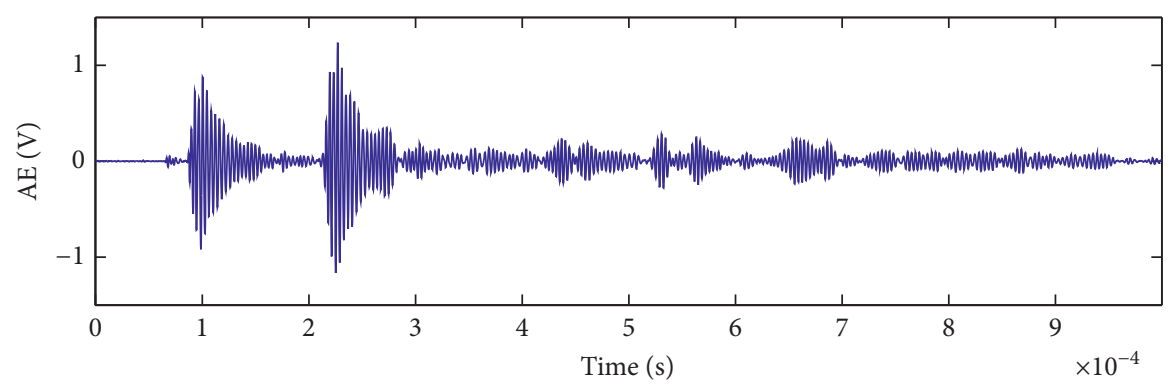

(a)

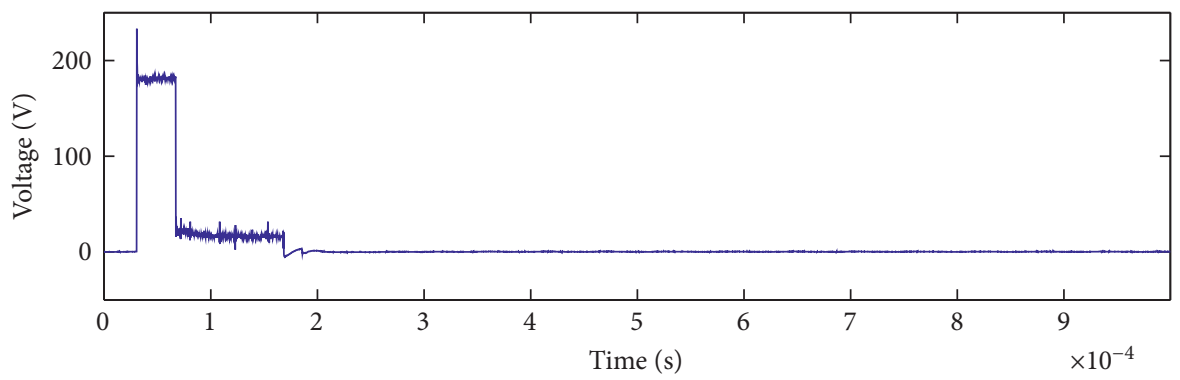

(b)

FIGURE 7: AE wave generated by discharge in a water-based emulsion.

wave, shown as the second peak in Figure 7. This result is consistent with Tamura's test result of the force change process during discharge [22].

For the discharge in a water-based emulsion, a fierce pressure fluctuation happened after the discharge process, which is the most significant difference compared to discharge in air and kerosene. The sharp pressure fluctuation would promote the melted material to be ejected from the workpiece surface and conducive to the material removal process.

In order to make a comparison for $\mathrm{AE}$ wave generated by discharge in kerosene and water-based emulsion, the peak amplitude and duration time of $\mathrm{AE}$ wave were measured for 100 consecutive discharges, respectively. The peak amplitude and duration time comparison result is shown in Figure 8. It can be noticed that the $\mathrm{AE}$ wave generated by discharge in 


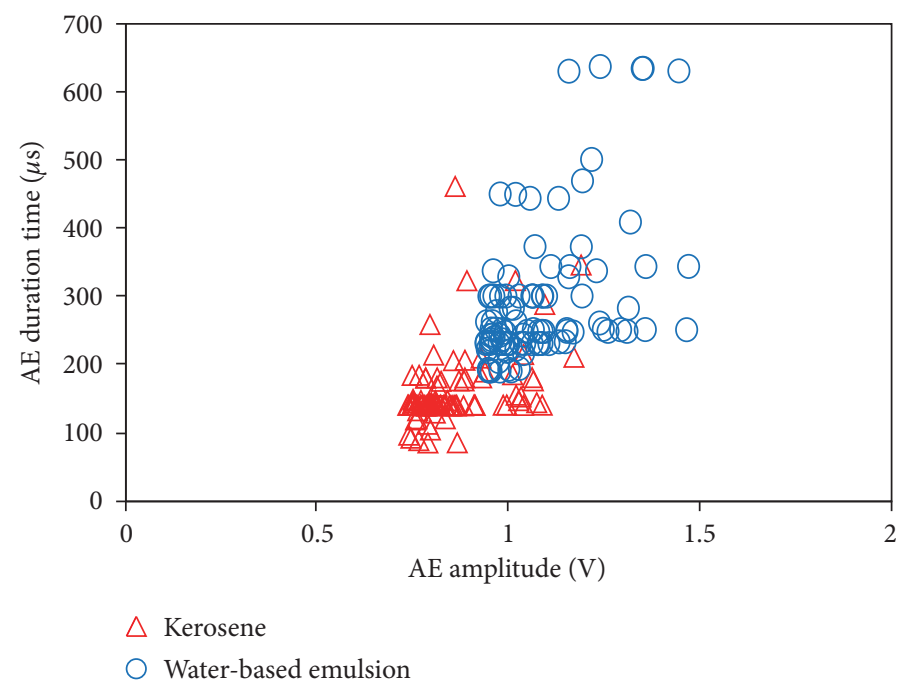

FIgURE 8: Comparison of AE duration and amplitude for discharge in kerosene and water-based emulsion. Characteristic analysis for discharge in water-based emulsion.

water-based emulsion has a higher peak amplitude and longer duration time than that in kerosene. It indicated that discharge in water-based emulsion would cause a more fierce pressure oscillation process and last a longer time than that in kerosene, which would be more conducive to the material removal process. In accordance with the result of Figures 2 and 3, discharge in water-based emulsion could get higher MRR with a rougher surface as a result of the fierce pressure oscillation process in the discharge gap.

In order to study the details of material removal for discharge in water-based emulsion, the discharge process was divided into 4 phases based on the expansion and contraction process of gas bubble surrounding discharge plasma. The process of each phase is illustrated in Figure 9.

Phase 1, discharge delay process, as shown in Figure 9(a): during this process, tool electrode moves toward the workpiece. The conductive microparticles in the water-based emulsion move toward the area where the electrical field is strong. Owing to the conductivity of the water-based emulsion, a large number of bubbles will be generated on the surface of the tool electrode and the workpiece by electrolysis reaction. The bubbles absorbed on the cathode surface are hydrogen, and the bubbles absorbed on anode surface are oxygen. The bubbles adsorbed on the electrode and workpiece surface improves the insulation effect between electrode and workpiece, which is beneficial to the discharge breakdown process. This process makes the discharge in water-based emulsion different from that in kerosene.

Phase 2, discharge breakdown process, as shown in Figure 9(b): when the interelectrode electric field strength reaches the water-based emulsion breakdown strength, the electrons on the surface of the cathode will have a field emission effect. The electrons will be accelerated and moving toward the anode, and its energy is enhanced during this process. When the electrons accumulated sufficient energy, the collision of the high-speed moving electrons in the water-based emulsion will cause the water-based emulsion to be vaporized and ionized. As a result, the number of electrons increased sharply. During this process, the diameter of the plasma column increases from the cathode to anode, as shown in Figure 9(b). (1). Owing to the collision of highspeed electrons, the material on the anode surface will be melted and vaporized. The water-based emulsion on the electrons moving path will be ionized, forming a discharge plasma channel between the electrode and workpiece as shown in Figure 9(b). (2). A strong AE wave is generated by this process.

Phase 3, discharge maintenance process, as shown in Figure 9(c): with power supply, the plasma channel will maintain at a high temperature. The water-based emulsion surrounding the plasma channel will be vaporized by the high temperature, forming a high-pressure bubble around the channel. The expansion and contraction process of the bubble will cause the pressure oscillation in the gap, which will generate an AE wave. The AE wave will fluctuate with the pressure oscillation of the bubble. During the discharge maintenance process, the hightemperature plasma column transfers energy to the electrodes and workpiece surface, which will cause the material on the electrode and workpiece surface to be melted and part of them even vaporized.

Phase 4, discharge suspending process, as shown in Figure 9(d): at the end of the discharge, the pulse power supply stopped the energy supply, and the high-temperature plasma column could not be maintained. Hydrogen and oxygen contained in the bubble surrounding plasma column will cause an explosion effect. The gas bubble will be destroyed quickly owing to no continuous energy supply available. The drastic change of pressure will cause a cavitation effect in the gap, which generates a strong $\mathrm{AE}$ wave and is helpful to 


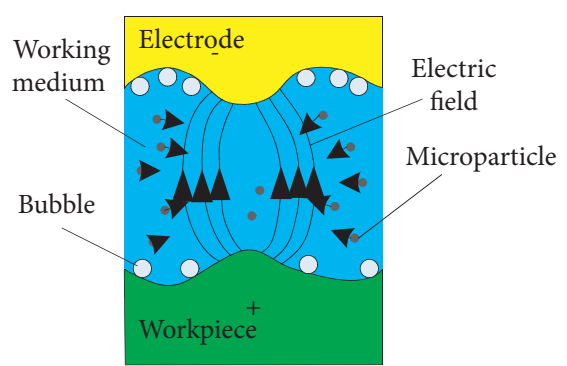

(1) Beginning of discharge delay

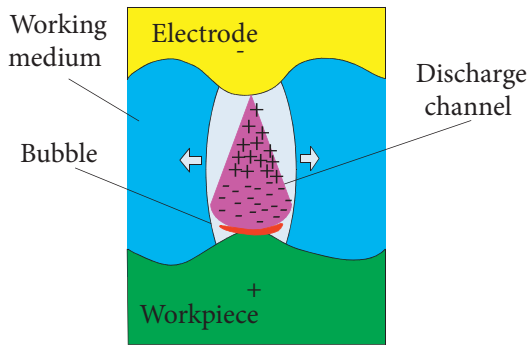

(1) Discharge breakdown moment

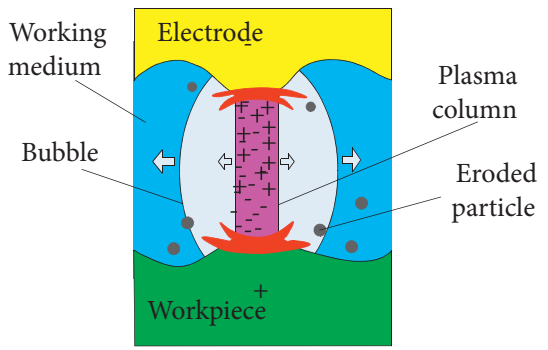

(1) Beginning of stable discharge

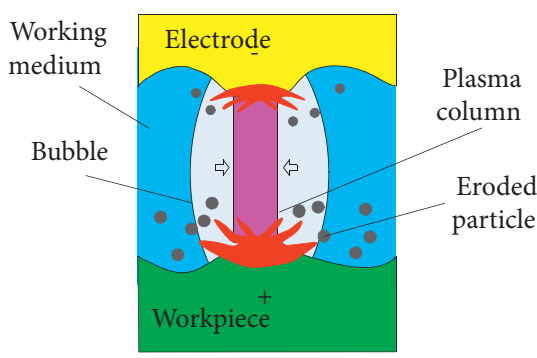

(1) Shrink of discharge channel

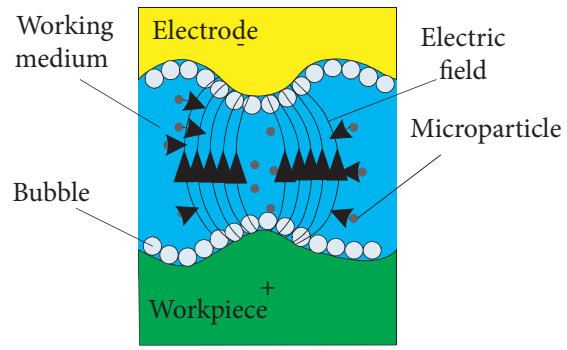

(2) End state of discharge delay

(a)

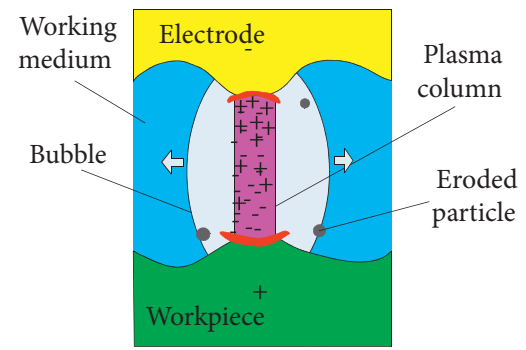

(2) Stable discharge channel

(b)

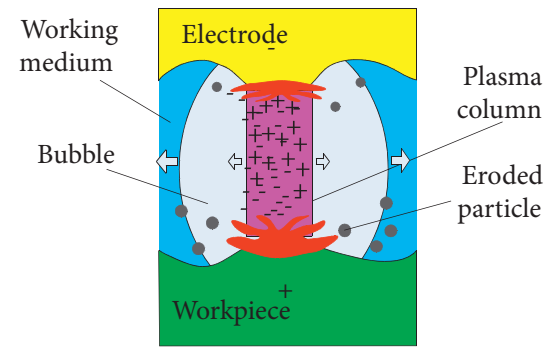

(2) Continuing of stable discharge

(c)

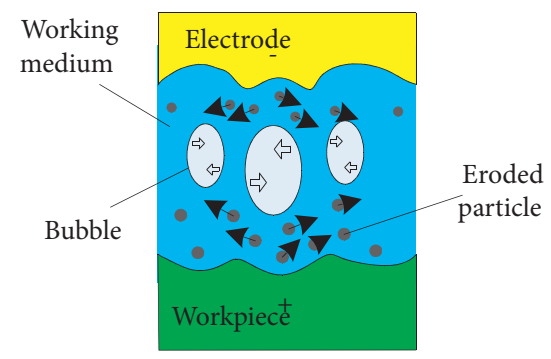

(2) After gas bubble collapse

(d)

FiguRE 9: The discharge process in water-based emulsion: (a) discharge delay process in the gap; (b) discharge breakdown process; (c) gap situations during stable discharge; and (d) gap situations after discharge.

remove the melted materials out of the discharge gap. This phenomenon is the most significant difference for the discharge process in the water-based emulsion compared to that in kerosene.

\section{Conclusions}

This research focused on studying the discharge process in different working mediums using the AE sensor. The details of the discharge process were discussed in accordance with the collected AE wave. Based on the experimental results, the main conclusions are drawn as follows:

(1) The working medium has an effect on the material removal process by influencing the pressure oscillation of gas bubble surrounding the discharge plasma during the discharge process, which made the MRR and surface topography in different working mediums different. 
(2) According to the frequency domain distribution analysis, the expansion and contraction frequency of gas bubble surrounding the discharge plasma was influenced by the working medium.

(3) Compared to discharge in kerosene, the discharge in water-based emulsion would generate a $\mathrm{AE}$ wave with a higher peak amplitude and longer duration time. Discharge in water-based emulsion would get a higher MRR with a rougher surface as a result of the fierce pressure oscillation process in the discharge gap.

(4) For EDM machining in the water-based emulsion, the cavitation effect at the end of the discharge process made the generated $\mathrm{AE}$ wave have a longer duration time with two peaks compared to that in kerosene, which was conducive to removing the melted materials from the workpiece surface.

\section{Data Availability}

The data used to support the findings of this study are available from the corresponding author upon request.

\section{Conflicts of Interest}

The authors declare that they have no conflicts of interest.

\section{Acknowledgments}

This work was supported by the Fundamental Research Funds for the Central Universities (no. 2572017BB06).

\section{References}

[1] S. Zhang, W. Zhang, Y. Liu, F. Ma, C. Su, and Z. Sha, "Corrigendum to "study on the gap flow simulation in EDM small hole machining with Ti alloy,"” Advances in Materials Science and Engineering, vol. 2017, p. 1, Article ID 7961763, 2017.

[2] C. Wang and Z. Qiang, "Comparison of micro-EDM characteristics of inconel 706 between EDM oil and an Al powder-mixed dielectric," Advances in Materials Science and Engineering, vol. 2019, Article ID 5625360, 11 pages, 2019.

[3] M. Kunieda, B. Lauwers, K. P. Rajurkar, and B. M. Schumacher, "Advancing EDM through fundamental insight into the process," Cirp Annals-Manufacturing Technology, vol. 54, no. 2, pp. 599-622, 2005.

[4] V. Prakash, P. Kumar, P. Singh, M. Hussain, A. Das, and S. Chattopadhyaya, "Micro-electrical discharge machining of difficult-to-machine materials: a review," Proceedings of the Institution of Mechanical Engineers, Part B: Journal of Engineering Manufacture, vol. 233, no. 2, pp. 339-370, 2017.

[5] M. B. Ndaliman, A. A. Khan, and M. Y. Ali, "Influence of dielectric fluids on surface properties of electrical discharge machined titanium alloy," Proceedings of the Institution of Mechanical Engineers, Part B: Journal of Engineering Manufacture, vol. 227, no. 9, pp. 1310-1316, 2013.

[6] K.-H. Tseng, H.-L. Lee, C.-Y. Liao, K.-C. Chen, and H.-S. Lin, "Rapid and efficient synthesis of silver nanofluid using electrical discharge machining," Journal of Nanomaterials, vol. 2013, Article ID 174939, 6 pages, 2013.

[7] A. A. A. Aliyu, A. M. Abdul-Rani, T. L. Ginta et al., "A review of additive mixed-electric discharge machining: current status and future perspectives for surface modification of biomedical implants," Advances in Materials Science and Engineering, vol. 2017, Article ID 8723239, 23 pages, 2017.

[8] W. Kong and X. Zhu, "Motion process research of cavitation bubble of ultrasonic electrical discharge machining," Journal of Physics: Conference Series, vol. 1176, Article ID 052033, 2019.

[9] M. Hadad, L. Q. Bui, and C. T. Nguyen, "Experimental investigation of the effects of tool initial surface roughness on the electrical discharge machining (EDM) performance," International Journal of Advanced Manufacturing Technology, vol. 95, no. 5-8, pp. 2093-2104, 2017.

[10] T. Kitamura, M. Kunieda, and K. Abe, "High-speed imaging of EDM gap phenomena using transparent electrodes," Procedia CIRP, vol. 6, pp. 314-319, 2013.

[11] T. Kitamura, M. Kunieda, and K. Abe, "Observation of relationship between bubbles and discharge locations in EDM using transparent electrodes," Precision Engineering, vol. 40, pp. 26-32, 2015.

[12] H. P. Schulze, G. Wollenberg, K. Mecke, and H. J. Trautmann, "Propagation of gas bubbles at spark erosion in small working gaps," in Proceedings of the 8th International Conference on Properties and Applications of Dielectric Materials, pp. 665668, Bali, Indonesia, June 2006.

[13] H. P. Schulze, G. Wollenberg, S. Matzen, and K. Mecke, "Origins of gas bubbles in a small work gap during the microEDM," in Proceedings of the 15th International Symposium on Electromachining, pp. 217-220, Pittsburgh, PA, USA, April 2007.

[14] S. Hayakawa, Y. Sasaki, F. Itoigawa, and T. Nakamura, "Relationship between occurrence of material removal and bubble expansion in electrical discharge machining," Procedia CIRP, vol. 6, pp. 174-179, 2013.

[15] J. Wang, F. Han, G. Cheng, and F. Zhao, "Debris and bubble movements during electrical discharge machining," International Journal of Machine Tools and Manufacture, vol. 58, pp. 11-18, 2012.

[16] F. Klocke, M. Mohammadnejad, M. Zeis, and A. Klink, "Potentials of the phase field approach for modeling modifications in material microstructure during electrical discharge machining," Procedia CIRP, vol. 42, pp. 703-708, 2016.

[17] S. Zhang, W. Zhang, P. Wang et al., "Simulation of material removal process in EDM with composite tools," Advances in Materials Science and Engineering, vol. 2019, Article ID 1321780, 11 pages, 2019.

[18] X. Yang, J. Guo, X. Chen, and M. Kunieda, "Molecular dynamics simulation of the material removal mechanism in micro-EDM," Precision Engineering, vol. 35, no. 1, pp. 51-57, 2011.

[19] Y. Z. Zhang, Y. H. Liu, R. J. Ji, C. Zheng, Y. Shen, and X. L. Wang, "Transient dynamics simulation of the electrical discharge-generated bubble in sinking EDM," International Journal of Advanced Manufacturing Technology, vol. 68, no. 5-8, pp. 1707-1715, 2013.

[20] H. Dong, Y. Liu, M. Li et al., "Experimental investigation of water-in-oil nanoemulsion in sinking electrical discharge machining," Materials and Manufacturing Processes, vol. 34, no. 10, pp. 1129-1135, 2019.

[21] Y. Zhang, Y. Liu, Y. Shen, R. Ji, X. Wang, and Z. Li, "Diesinking electrical discharge machining with oxygen-mixed water-in-oil emulsion working fluid," Proceedings of the 
Institution of Mechanical Engineers, Part B: Journal of Engineering Manufacture, vol. 227, no. 1, pp. 109-118, 2012.

[22] T. Tamura and Y. Kobayashi, "Measurement of impulsive forces and crater formation in impulse discharge," Journal of Materials Processing Technology, vol. 149, no. 1-3, pp. 212216, 2004.

[23] W. Natsu, M. Shimoyamada, and M. Kunieda, "Study on expansion process of EDM arc plasma," JSME International Journal Series C, vol. 49, no. 2, pp. 600-605, 2006.

[24] A. Kojima, W. Natsu, and M. Kunieda, "Spectroscopic measurement of arc plasma diameter in EDM," CIRP Annals, vol. 57, no. 1, pp. 203-207, 2008.

[25] C. Qu, W. Natsu, and M. Kunieda, "Clarification of EDM phenomena by spectroscopic analysis," Journal of Advanced Mechanical Design, Systems, and Manufacturing, vol. 6, no. 6, pp. 908-915, 2012.

[26] X. L. Xu and Z.-Z. Zhang, "Acoustic emission and damage characteristics of granite subjected to high temperature," Advances in Materials Science and Engineering, vol. 2018, Article ID 8149870, 12 pages, 2018.

[27] Z. Wen, X. Wang, L. Chen, G. Lin, and H. Zhang, "Size effect on acoustic emission characteristics of coal-rock damage evolution," Advances in Materials Science and Engineering, vol. 2017, Article ID 3472485, 8 pages, 2017.

[28] F. H. Çakir and O. N. Çelik, "Improvement of the machinability of Ti-6Al-4V alloy wire electro discharge machining with cryogenic treatment," Metals and Materials International, vol. 2, 2020.

[29] F. H. Çakir and O. N. Çelik, "Tribological properties of cryotreated and aged Ti6Al4V alloy," Transactions of the Indian Institute of Metals, vol. 73, no. 3, pp. 799-809, 2020. 